\title{
ELASTIC INTRAMEDULLARY NAILING IN PAEDIATRIC DIAPHYSEAL FOREARM FRACTURES
}

\author{
Amit Kumar Chaurasia1, Pradeep Kumar Verma²
}

${ }^{1}$ Associate Professor, Department of Orthopaedics, S.S. Medical College \& SGM Hospital, Rewa.

${ }^{2}$ Assistant Professor, Department of Orthopaedics, S.S. Medical College \& SGM Hospital, Rewa.

ABSTRACT

\section{BACKGROUND}

The majority of paediatric diaphyseal fractures of the radius and ulna can be treated well with closed reduction and cast immobilization. The most common indications for surgery are failure of closed reduction, open fractures and fracture instability. Over recent years the forearm fractures are increasingly being treated with intramedullary elastic nails to prevent displacement during the healing phase.

\section{OBJECTIVE}

Recently, there has been an increased interest in determining which method provides superior results, but the optimal treatment remains controversial. This article analyses the results of 22 diaphyseal forearm fractures in children who underwent flexible intramedullary nail fixation.

\section{MATERIAL AND METHODS}

A prospective study was conducted from January 2012 to December 2015, on the patients with both-bone forearm fractures. Inclusion criteria of study were age between 5 and 15 years, closed displaced fractures, unacceptable closed reduction and open displaced fractures (Type 1 and 2). A single nail was used for each forearm bone.

\section{RESULTS}

The results of the 22 patients (16 males and 6 females) who were treated in our institution with closed elastic nails were studied systematically with a follow-up period from 4 months to 15 months. At follow-up clinics, all patients went on to osseous union and regained a full range of movement after rehabilitation. There were no cases of delayed union, non-union or mal-union. All implants were routinely removed within average time of 8 months (Range: 6-10) after nailing. There were no complications after implant removal in our patients.

\section{CONCLUSION}

Conservative management is still the first line of treatment for paediatric forearm fractures, especially in children less than 10 years old. Treatment with an elastic intramedullary nail is indicated for unstable, irreducible or open fractures when non-operative management fails.

\section{KEYWORDS}

Elastic Intramedullary Nailing, Paediatric Forearm Fractures, Diaphyseal Fracture.

HOW TO CITE THIS ARTICLE: Chaurasia AK, Verma PK. Elastic intramedullary nailing in paediatric diaphyseal forearm fractures J. Evolution Med. Dent. Sci. 2016;5(21):1075-1079, DOI: 10.14260/jemds/2016/249

\section{INTRODUCTION}

Paediatric diaphyseal fractures of the radius and ulna are the third most common fracture in the paediatric population and account for $13-40 \%$ of all paediatric fractures.1,2 The majority of these fractures can be treated well with closed reduction and cast immobilization due to the unique property of the growth potential of the immature bones. Nevertheless, there is a subset of patients in whom surgical intervention is indicated. The most common indications for surgery are failure of closed reduction, open fractures and fracture instability. In these situations, if left untreated malunion is more likely to occur, which will disturb the function of the upper extremities. ${ }^{3,4}$ Over recent years the use of elastic stable intramedullary nails has dramatically increased with the introduction of a variety of nails for paediatric fractures.

Financial or Other, Competing Interest: None.

Submission 14-02-2016, Peer Review 26-02-2016,

Acceptance 29-02-2016, Published 11-03-2016.

Corresponding Author:

Dr. Amit Kumar Chaurasia,

Chaurasia Hospital, Jail Road

Rewa-486001,

Madhya Pradesh.

E-mail:amit140975@yahoo.com

DOI: $10.14260 /$ jemds $/ 2016 / 249$
The forearm fractures, especially with both bones involved, are increasingly being treated with intramedullary elastic nails to prevent displacement during the healing phase. $5,6,7$ Elastic intramedullary nails were originally developed in the early 1980s by surgeons in Nancy, France. ${ }^{8}$ Recently, there has been an increased interest in determining which method provides superior results, but the optimal treatment remains controversial. ${ }^{9}$ This article analyses the results of 22 diaphyseal forearm fractures in children who underwent flexible intramedullary nail fixation.

\section{MATERIAL AND METHODS}

A prospective study was conducted in the Department of Orthopaedics, S.S. Medical College Hospital, Rewa, from January 2012 to December 2015, on the patients with bothbone forearm fractures in the age group of 5 years - 15 years of either sex. An unacceptable alignment was defined as less than $50 \%$ cortical contact between the fragments and greater than $10^{\circ}$ of angulation in either the sagittal or coronal plane. Inclusion criteria of study were age between 5 and 15 years, closed displaced fractures, unacceptable closed reduction and open displaced fractures (Type 1 and 2).

Exclusion criteria were age beyond range of 5 to 15 , greenstick fractures, undisplaced fractures, acceptable reduction and open fractures (Type 3). 
A single nail was used for each forearm bone. Generally, a nail of 2.0 to $2.5 \mathrm{~mm}$ diameter was used. The nail in the radius was inserted just proximal to the physis on the radial border. An alternate point of entry in some cases was dorsally adjacent to Lister's tubercle. The nail in the ulna was inserted just distal to the physis on the radial border. The protruding parts of the nails were cut keeping a small part outside. All patients were immobilized postoperatively in an aboveelbow plaster slab for 4 weeks. Follow-up examination of patients included progress of fracture healing, Range of Motion (ROM), angular deformities and measurement of limb length.

\section{RESULTS}

The results of the 22 patients (16 males and 6 females) who were treated in our institution were studied systematically with a follow-up period from 4 months to 15 months. In our series, the range of age of patients was from 5 years to 15 years with an average of 8 years. Out of 22 patients, 14 children (66\%) had injury on the right side. In 8 patients $(36 \%)$, the mechanism of injury was due to motor vehicle accident, $4(18 \%)$ were due to fall from height, while remaining $10(45 \%)$ were due to fall while playing. Closed reduction and closed elastic nail fixation was successful in 18 cases including 16 both-bone fractures and 2 single-bone fracture. Open reduction with a mini-open procedure was carried out in 4 fractures that affected both-bone fractures in 3 patients. All of the fractures healed within an average of 7 weeks (Range: 6-9).

No non-unions, delayed unions or malunion were found. There was no notable difference in the healing time either for fractures of both bones or for isolated radial or ulnar fractures. All implants were routinely removed under intravenous sedation. The average time for removal of the implants in this study was 8 months (Range: 6-10). There were no complications after implant removal in our patients. Complications as a result of the procedure included: one neuropraxia involving the superficial radial nerve, which resolved after several weeks with no long-term complication. One patient also presented with protrusion of the wires through the skin, although they had been buried during the procedure. These nails required removal 2-3 weeks prior to the planned date of removal.

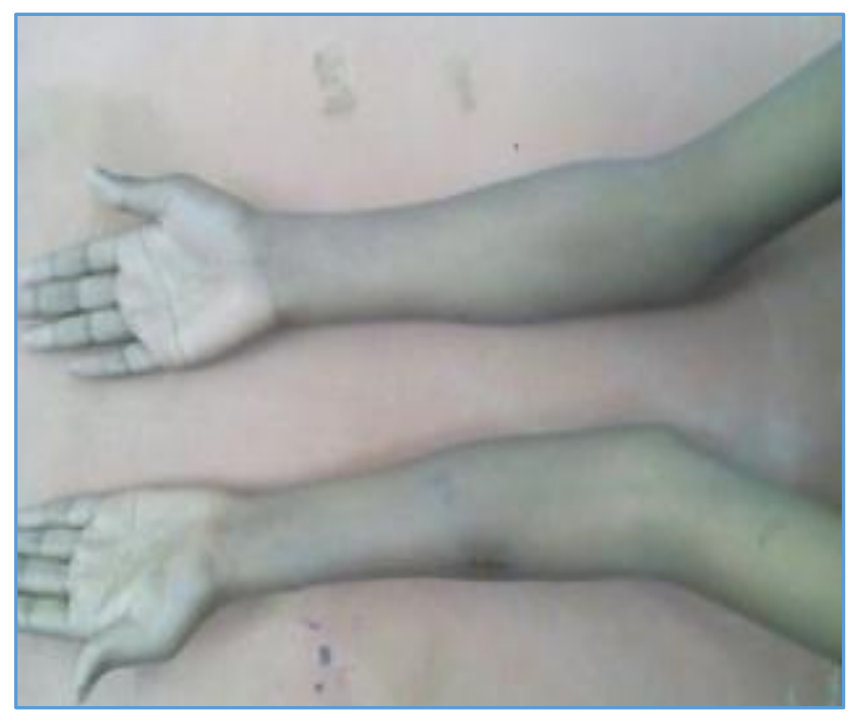

Fig. 1: Follow-up Function 2

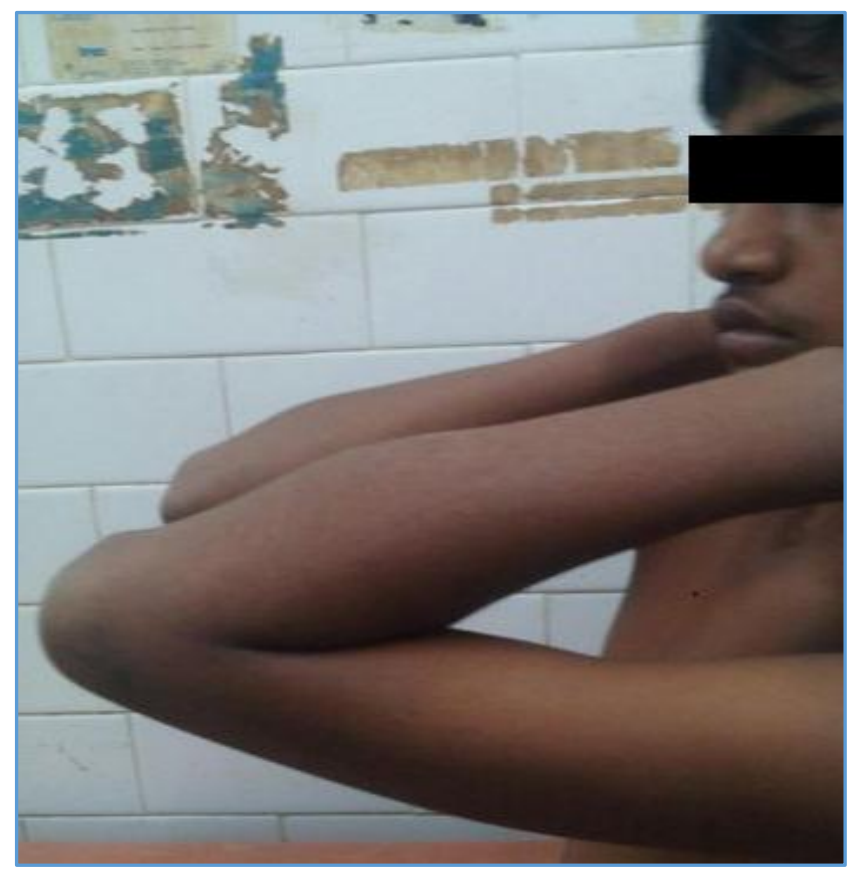

Fig. 2: Follow-up Function

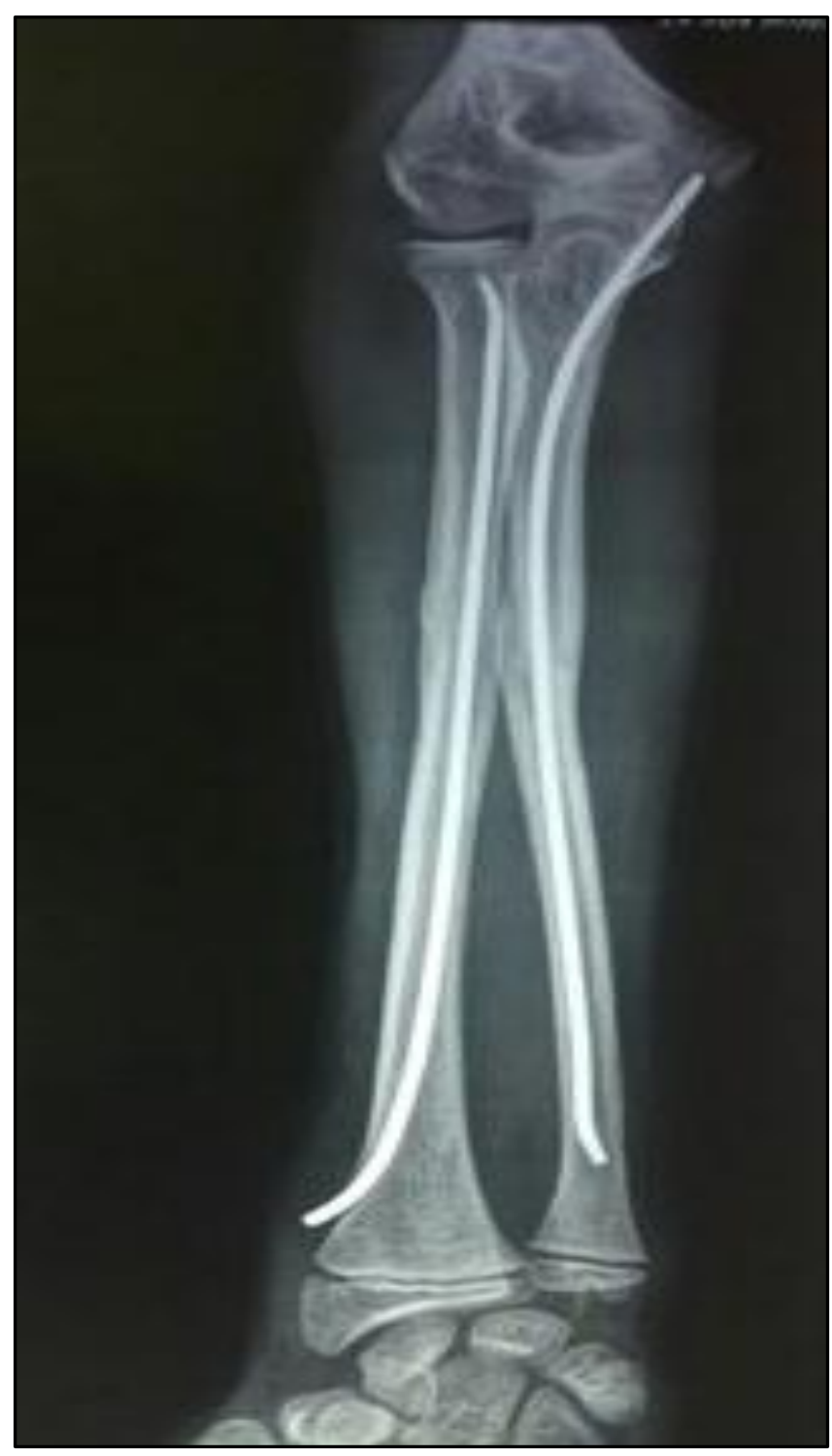

Fig. 3: Follow-up X-ray at 6 months 

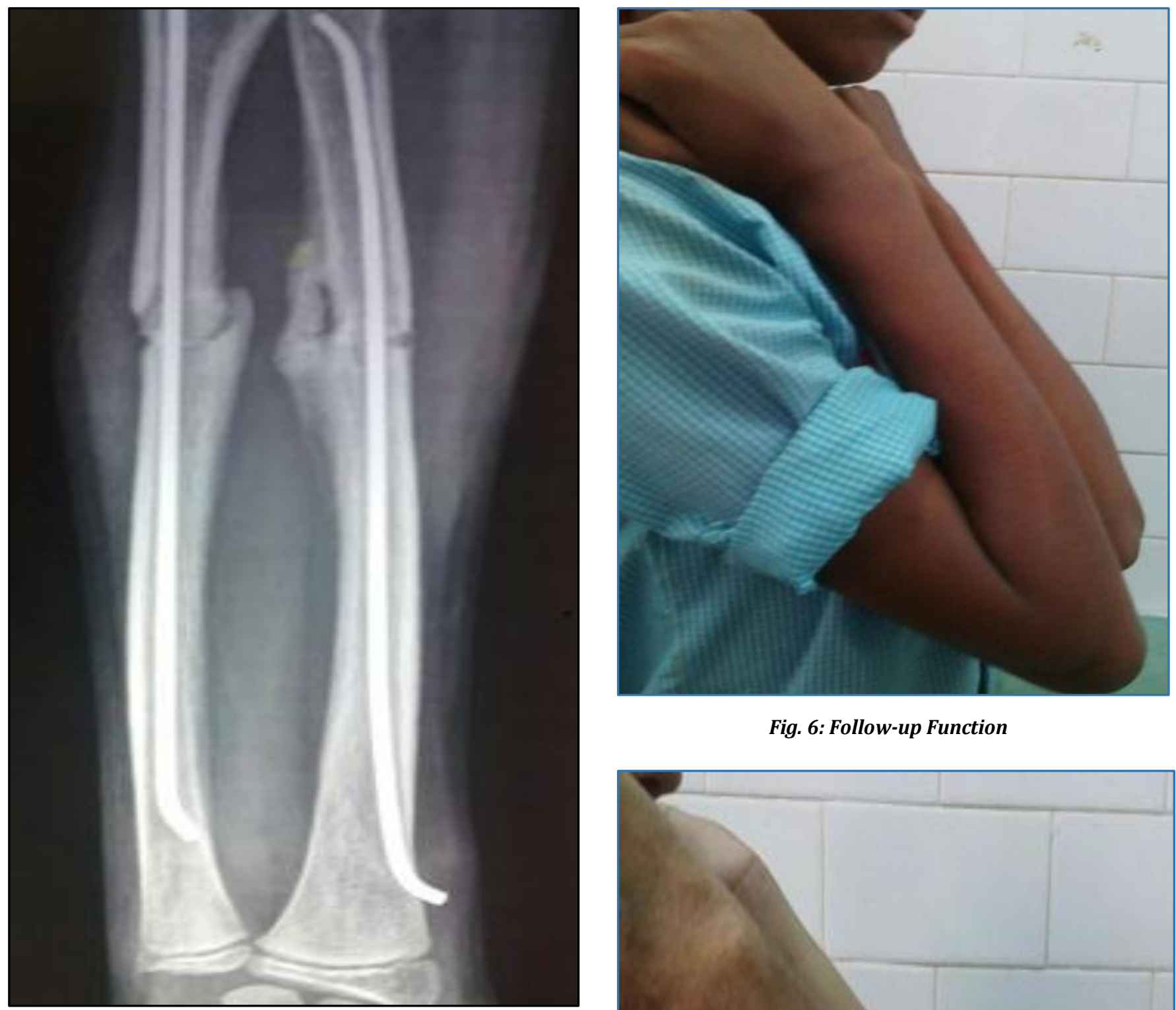

Fig. 6: Follow-up Function

Fig. 4: Follow-up X-ray
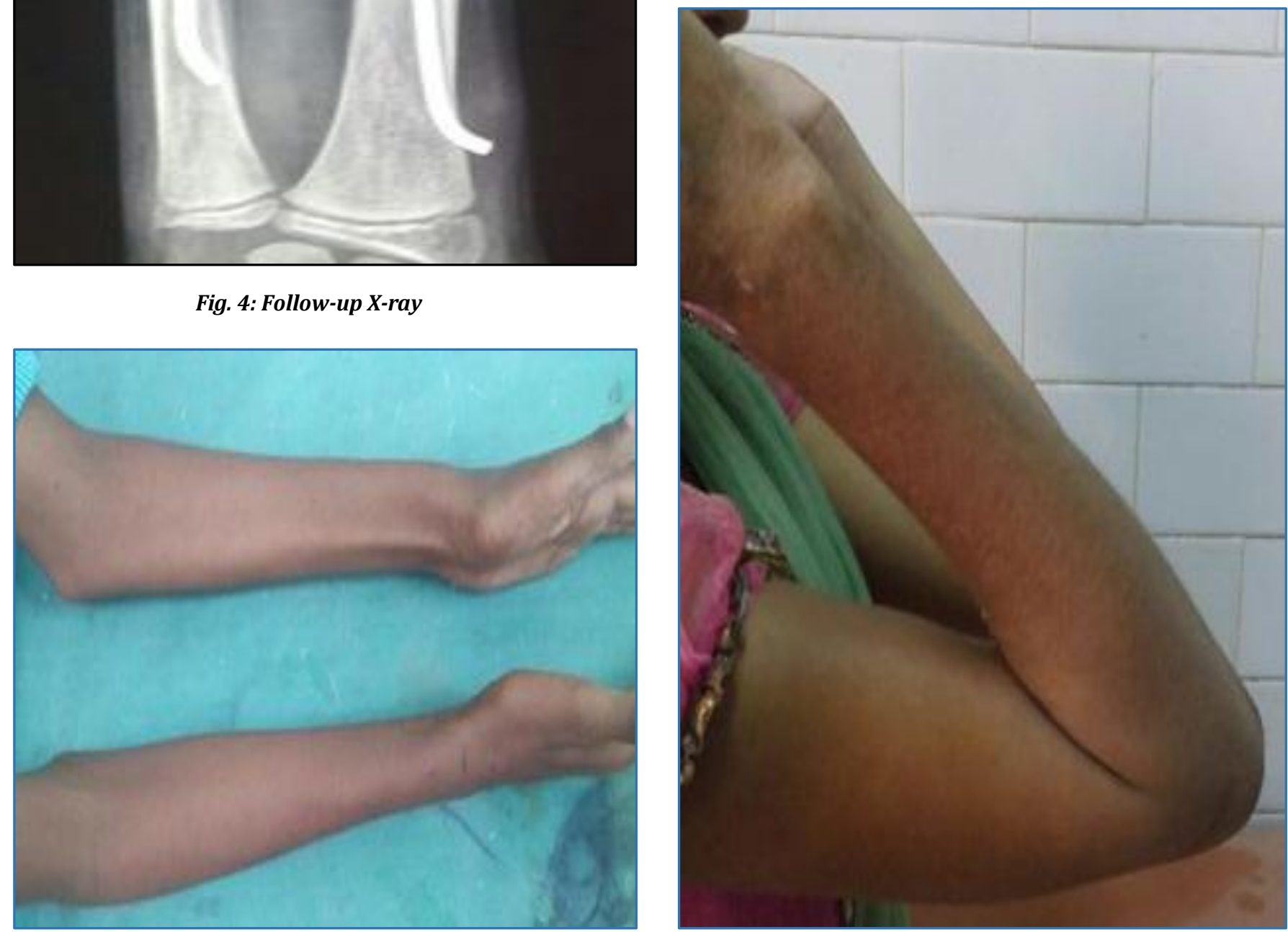

Fig. 5: Follow-up Function 2

Fig. 7: Function at 3 months 


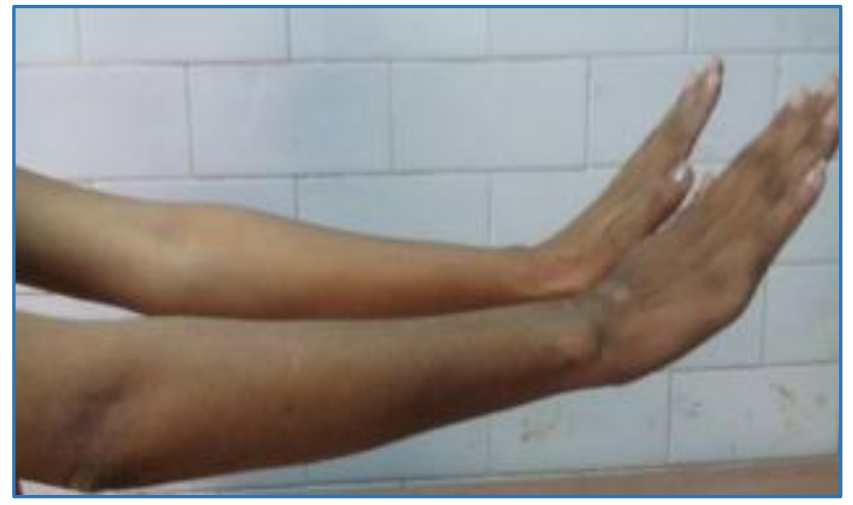

Fig. 8: Function at 3 months
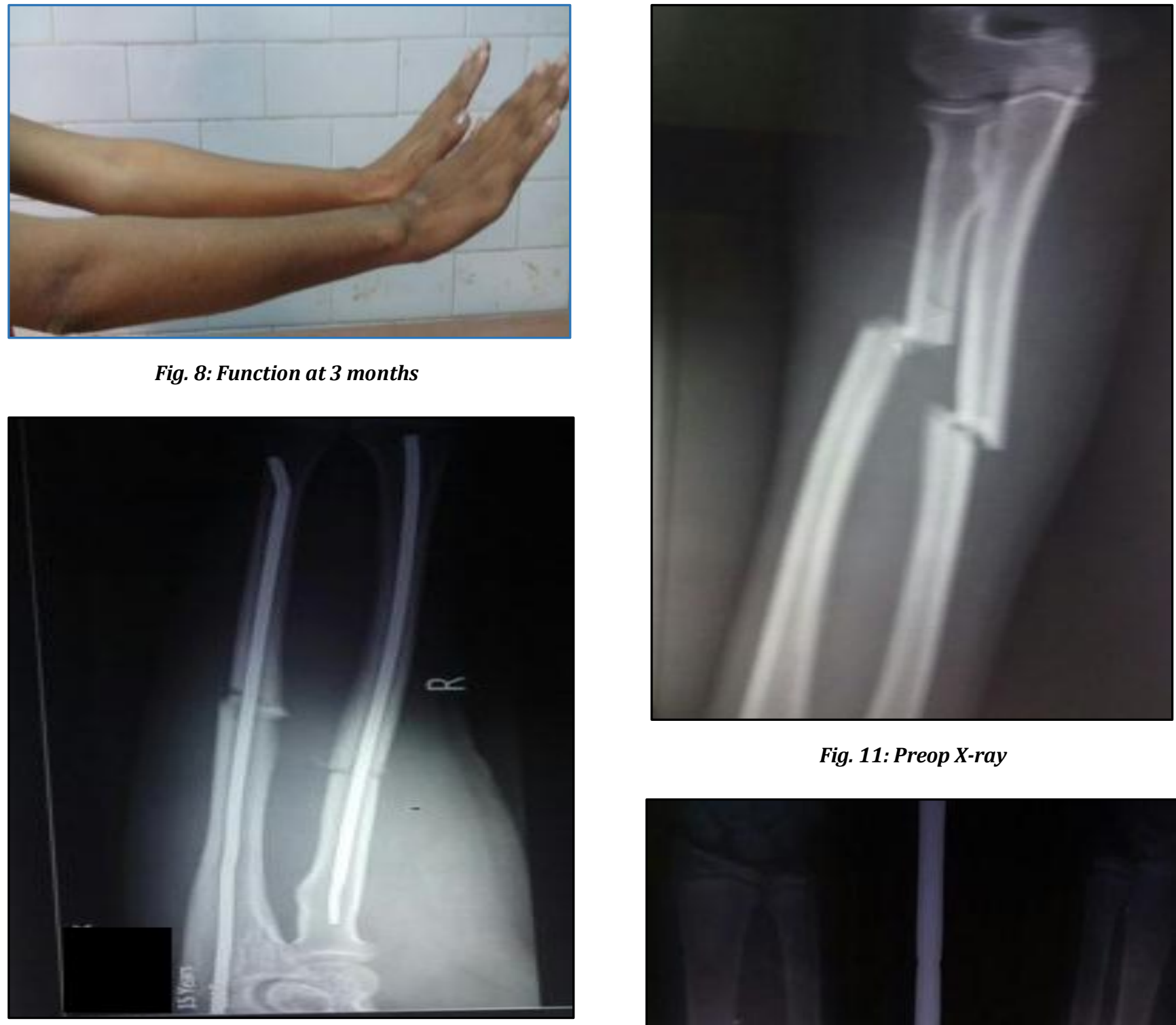

Fig. 11: Preop X-ray

Fig. 9: Postop X-ray at 3 months
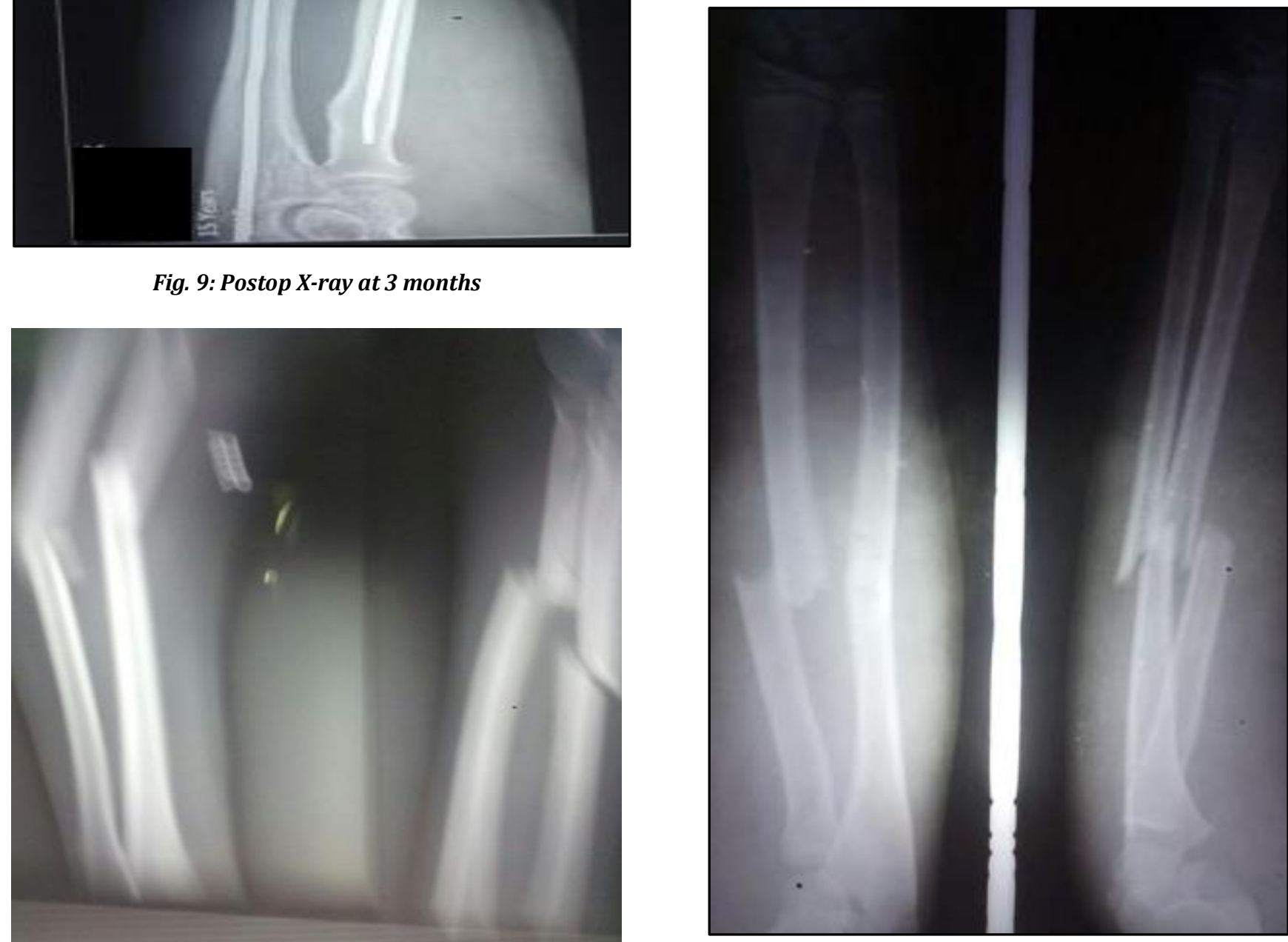

Fig. 10: Preop Radiograph

Fig. 12: Preop X-ray 


\section{DISCUSSION}

The gold standard for paediatric forearm fractures remains closed reduction and casting. ${ }^{2}$ Previous papers have shown excellent results in children under the age of ten years with closed treatment with poorer results and increased incidence of re-displacement in children over the age of ten years. ${ }^{6}$ Elastic intramedullary nails are now commonly used for the treatment of unstable type paediatric forearm fractures. This technique offers stable fixation without disturbance of the periosteal blood supply and fracture hematoma, which contributes to fracture healing. This technique also allows for micro-motion at fracture site to stimulate the callus formation to bridge the fracture gaps. Kang et al. evaluated 90 children treated with intramedullary nailing and reported good results and patient outcomes. ${ }^{10}$ The average patient age was 8.4 years with a follow-up of 6.6 months; $86 \%$ (77/90) of the patients had both-bone fractures and 9\% (8/90) had open fractures.

Ten were treated with flexible nailing after failed closed management; the remaining fractures were irreducible or considered unstable; $44 \%$ of the fractures required limited open reduction to allow passage of the nail and to obtain satisfactory alignment. All fractures healed by 3 months; $84 \%$ (76/90) of their patients had outcomes that were excellent (Equal motion between sides) or good outcomes (Less than 20-degree loss of motion versus uninjured arm). Based on their findings, the authors recommended the use of intramedullary nailing for treatment of forearm fractures not amenable to closed reduction or after failed closed reduction. Intramedullary fixation of forearm fractures has been reported unsuccessful in the adult literature and only recently the technique has been adapted to the management of forearm fractures in children.7,11,12,13 Amit et al. reported 20 unstable diaphyseal fractures of the forearm in adolescent patients treated with closed intramedullary nailing.

All fractures healed within 6 weeks. There was no crossunion, non-union, infection or refracture. Amit et al. favoured that technique rather than plate fixation because of the appropriate reduction, reduced complication rate, negligible cosmetic defect and the ability to perform rod removal under local anaesthesia. ${ }^{11}$ In the paediatric patient, non-union has not been reported in the literature and good/excellent functional results are reported in nearly $95 \%$ of cases. $12,13,14$ These excellent clinical results support the use of elastic intramedullary nails in the operative treatment of forearm fractures in the paediatric patient.

\section{CONCLUSION}

Closed reduction and Elastic nail fixation was successful in 18 cases including 16 both-bone fractures and 2 single-bone fracture. Open reduction with a mini-open procedure was carried out in 4 fractures that affected both bones in 3 patients. Bone union was achieved in all patients at an average of 7 weeks without any significant complications. Conservative management is still the first line of treatment for paediatric forearm fractures, especially in children less than 10 years old. Treatment with an elastic intramedullary nail is indicated for unstable, irreducible or open fractures, when non-operative management fails.

\section{REFERENCES}

1. Cheng JC, Ng BK, Ying SY, et al. A 10-year study of the changes in the pattern and treatment of 6,493 fractures. J Pediatr Orthop 1999;19(3):344-50.

2. Jones K, Weiner DS. The management of forearm fractures in children: a plea for conservatism. J Pediatr Orthop 1999;19(6):811-5.

3. Daruwalla JS. A study of radioulnar movements following fractures of the forearm in children. Clin Orthop Relat Res 1979;139:114-120.

4. Fuller DJ, McCullough CJ. Malunited fracture of the forearm in children. J Bone Joint Surg Br 1982;64(3):364367.

5. Huber RI, Keller HW, Iluber PM, et al. Flexible intramedullary nailing as fracture treatment in children. J Pediatr Orthoped 1996;16(5):602-605.

6. Kay MD, Smith C, Oppenheim WL. Both-bone midshaft forearm fractures in children. J Pediatr Orthoped 1986;6(3):306-310.

7. Lascombes P, Prevot J, Ligier JN, et al. Elastic stable intramedullary nailing in forearm shaft fractures in children: 85 cases. J Pediatr Orthoped 1990;10:167-171.

8. Barry M, Paterson JM. A flexible intramedullary nails for fractures in children. J Bone Joint Surg $\mathrm{Br}$ 2004;86(7):947-53.

9. Abraham A, Kumar S, Chaudhry S, et al. Surgical interventions for diaphyseal fractures of the radius and ulna in children. Cochrane Database Syst Rev 2011;9(11):CD007907.

10. Kang SN, Mangwani J, Ramachandran M, et al. Elastic intramedullary nailing of paediatric fractures of the forearm: a decade of experience in a teaching hospital in the united kingdom. J Bone Joint Surg Br 2011;93(2):2625.

11. Amit Y, Salai M, Chechik A, et al. Closing intramedullary nailing for the treatment of diaphyseal forearm fractures in adolescence: a preliminary report. J Pediatr Orthop 1985;5(2):143-6.

12. Smith H, Sage FP. Medullary fixation of forearm fractures. J Bone Joint Surg Am 1957;39(1):91-8.

13. Street DM. Intramedullary Forearm Nailing. Clin Orthop Relat Res 1986;212:219-30.

14. Cullen MC, Roy DR, Giza E, et al. Complications of intramedullary fixation of pediatric forearm fractures. J Pediatr Orthop 1998;18(1):14-21. 